\title{
Human-Centered Design and Sustainable Malaria Interventions
}

\author{
Michael Macdonald, ${ }^{a}$ Thomas Putzer ${ }^{b}$
}

Human-centered design provides a method to adapt malaria control interventions to be more closely aligned with a family's convenience, comfort, and personal lifestyle, enabling a broader and more sustained culture of access and use.

See related article by Kim.

For every complex problem there is an answer that is clear, simple, and wrong. - H.L. Mencken (paraphrased), Prejudices: Second Series (1920)

$\mathbf{T}$ he article by Kim et al.," "Using a human-centered design approach to determine consumer preferences for long-lasting insecticidal nets in Ghana," raises 2 important questions. First, can the public sector adapt long-lasting insecticidal net (LLIN) designs to the user's point of view rather than purely technical and cost considerations? Second, is it possible to segment the market for mosquito nets such that people who can afford to pay can acquire the nets they want commercially, while the public sector focuses limited resources on those most in need who are unable to afford to purchase nets?

These questions challenge current strategies on 2 counts. First, large international tenders for mosquito nets are awarded on unit costs that meet the minimum physical standards. Second, "universal coverage" demands that all persons living in malaria endemic areas receive a free standard net.

While treated mosquito nets can have a significant impact on malaria illness and death ${ }^{2}$ some estimate that, depending on the community being studied, as much as half of the households that receive nets do not use them for their intended purpose. ${ }^{3}$ Excessive heat and reduced airflow are often cited as barriers to LLIN use, ${ }^{4}$ with reduced air flow ranging from $55 \%$ to $71 \%$ in 1 study of 11 commercial nets. ${ }^{5}$ Imagine how much more impact LLINs could make if design changes to increase end-user compliance were addressed.

\footnotetext{
a Associate Editor, Global Health: Science and Practice Journal, and Consultant, Catonsville, MD, USA.

bSC Johnson \& Son, Inc., Racine, WI, USA.

Correspondence to Michael Macdonald (macdonaldm@macito.net).
}

Kim et al. explored the barriers to LLIN ownership and use among middle-class Ghanaians through a humancentered design (HCD) process that moves beyond the traditional public health tools of focus group discussions, household surveys, and trials of improved practices:

The result was a rich mix of data and the identification of key consumer insights regarding middle-class Ghanaians' perceptions of self, their behaviors and attitudes related to malaria prevention, and their use of LLINs.

The study found:

... in most accounts [free public-sector LLINs] were inconvenient, uncomfortable, and not aesthetically pleasing, thus they were undesirable to use.

Suggested changes to the standard LLINs included a more convenient way to hang the net, a more attractive silhouette, and a zipper for ease of entry and exit. Previous LLIN design work in Ghana included the addition of a solar-powered light and fan. ${ }^{6}$

HCD, the process described by Kim et al., is an iterative approach to generating solutions that are firmly rooted in people, developing empathy with the end-user, generating an abundance of ideas, building tangible prototypes, and iteratively co-creating with people again. Four lenses of user desirability, business viability, technical feasibility, and sustainability typically serve as a framework, focusing on optimizing for the user experience while ensuring viability at scale. HCD has become a key element in the Center for Innovation and Impact $^{7}$ at the United States Agency for International Development and at the Design for Health Initiative. ${ }^{8}$

The value of HCD for improving LLIN access and use is well recognized. However, the business viability of implementing HCD solutions that go beyond addressing only the technical feasibility is challenged by the current system. Currently, manufacturers are simply "vendors" rather than "partners," with a focus on manufacturing uniform LLINs, with minimum specifications at the 
lowest possible price, in order to secure large tenders for mass distribution. With the sole focus on price, limited opportunities exist for innovations that address barriers to use, such as convenience, comfort, and personal lifestyle. ${ }^{1}$

Ultimately, our hope is that, as HCD emphasizes, people impacted by malaria will be enabled to guide us according to their priorities, how they live, and what is important to them. In turn, we will have a chance to create sustainable solutions that fit their lifestyles and will prevent malaria.

With the uncertainty of future global health funding, ${ }^{9}$ it is more important than ever that we optimize available resources to segment LLIN delivery strategies - that is, facilitate the growth of a consumer market for those who can afford to make purchases and concentrate public health resources on those who cannot, offering a diversity of LLIN products that fit the needs and preferences of each.

In his commentary on the survival of the Global Fund to Fight AIDS, Tuberculosis and Malaria, Richard Horton notes ${ }^{10}$ :

There are, of course, other questions the Fund must consider. How far should it embrace the private sector? What is its strategy for middle-income countries? How can the Fund leverage domestic investments...?

In the context of LLIN distributions, this could be rephrased: How do we facilitate a commercial LLIN sector, what is the strategy for middleincome families, how can the Fund leverage consumer investments?

Kim et al. ${ }^{1}$ provide a step in that direction:

We have since shared our consumer insights and preliminary ideas for new design features with current manufacturers globally who supply LLINs in Ghana. We hope these partners take this information into consideration as they make decisions about current and future LLIN supply, demand, and marketing and will pursue pilot testing of new net designs for the private sector retail market in Ghana.
They conclude with a statement that seems to sometimes be lost on policy makers:

For a health technology such as the LLIN to produce a benefit, it has to be used.

There is much to be learned from consumer product development and HCD to solve complex problems for which we thought, with our hubris, we already knew the simple solution.

Competing Interests: None declared.

\section{REFERENCES}

1. Kim S, Piccinini D, Mensah E, Lynch M. Using a human-centered design approach to determine consumer preferences for long-lasting insecticidal nets in Ghana. Glob Health Sci Pract. 2019;7(2):160170. CrossRef

2. Malaria fact sheet. World Health Organization website. https:// www.who.int/en/news-room/fact-sheets/detail/malaria. Updated March 27, 2019. Accessed May 28, 2019.

3. Shah S. The Fever: How Malaria Has Ruled Humankind for 500,000 Years. New York: Farrar, Straus and Giroux; 2010.

4. Pulford J, Hetzel MW, Bryant M, Siba PM, Mueller I. Reported reasons for not using a mosquito net when one is available: a review of the published literature. Malar J. 2011;10:83. CrossRef. Medline

5. von Seidlein L, Ikonomidis K, Bruun R, et al. Airflow attenuation and bed net utilization: observations from Africa and Asia. Malar J. 2012;11:200. CrossRef. Medline

6. Jaeger MS, BriëtOJ, Keating J, et al. Perceptions on the effect of small electric fans on comfort inside bed nets in southern Ghana: a qualitative study. Malar J. 2016;15(1):580. CrossRef. Medline

7. Center for Innovation and Impact. 2019 Impact Brief. Washington, DC: United States Agency for International Development (USAID), Bureau for Global Health; 2019. https://www.usaid.gov/sites/ default/files/documents/1864/USAID_2019_CII_Lookbook web. pdf. Accessed May 28, 2019.

8. Design for Health website. https://www.designforhealth.org/. Accessed May 28, 2019

9. Coester K, Conron E, Heiberg D. 5 Things to know about US global health funding for FY 2020. Global Health NOW website. https://www.globalhealthnow.org/2019-05/5-things-knowabout-us-global-health-funding-fy-2020. Published May 6, 2019. Accessed May 28, 2019.

10. Horton R. Offline: can the Global Fund survive? Lancet. 2018;392 (10141):14. CrossRef. Medline

Cite this article as: Macdonald M, Putzer T. Human-centered design and sustainable malaria interventions. Glob Health Sci Pract. 2019;7(2):148-149. https://doi.org/10.9745/GHSP-D-19-00189

(C) Macdonald and Putzer. This is an open-access article distributed under the terms of the Creative Commons Attribution 4.0 International License (CC BY 4.0), which permits unrestricted use, distribution, and reproduction in any medium, provided the original author and source are properly cited. To view a copy of the license, visit http://creativecommons.org/licenses/by/4.0/. When linking to this article, please use the following permanent link: https://doi.org/10.9745/GHSP-D-19-00189 